\title{
Striatal astrocytes produce neuroblasts in an excitotoxic model of Huntington's disease
}

\author{
Giulia Nato ${ }^{1,2}$, Alessia Caramello 1,2 , Sara Trova ${ }^{1,2}$, Valeria Avataneo ${ }^{1,2}$, Chiara Rolando ${ }^{3}$, Verdon Taylor ${ }^{3}$, \\ Annalisa Buffo ${ }^{2,4}$, Paolo Peretto ${ }^{1,2, *, \ddagger}$ and Federico Luzzati ${ }^{1,2, *, \neq}$
}

\begin{abstract}
In the adult brain, subsets of astrocytic cells residing in well-defined neurogenic niches constitutively generate neurons throughout life. Brain lesions can stimulate neurogenesis in otherwise non-neurogenic regions, but whether local astrocytic cells generate neurons in these conditions is unresolved. Here, through genetic and viral lineage tracing in mice, we demonstrate that striatal astrocytes become neurogenic following an acute excitotoxic lesion. Similar to astrocytes of adult germinal niches, these activated parenchymal progenitors express nestin and generate neurons through the formation of transit amplifying progenitors. These results shed new light on the neurogenic potential of the adult brain parenchyma.
\end{abstract}

KEY WORDS: Neural stem cells, Parenchymal progenitors, Stem cell quiescence, Lesion-induced neurogenesis, Huntington's disease, Mouse

\section{INTRODUCTION}

In adult neurogenic niches, astrocytic cells produce neurons throughout life (Fuentealba et al., 2012). Parenchymal astrocytes can become neurogenic in vitro when isolated from the lesioned neocortex (Buffo et al., 2008; Sirko et al., 2013) or in vivo after overexpression of specific transcription factors (Niu et al., 2013). However, whether parenchymal astrocytes can spontaneously generate neurons in vivo is unclear (Dimou and Götz, 2014). The adult brain parenchyma has been generally considered gliogenic and not permissive for the activity of neuronal progenitors (Lim et al., 2000; Ninkovic and Götz, 2013; Shihabuddin et al., 2000). Nonetheless, examples of parenchymal neurogenesis are emerging (Bi et al., 2011; Luzzati et al., 2006, 2011b; Ohira et al., 2010). For instance, we previously demonstrated that clusters of proliferating cells with features of transient amplifying progenitors (TAPs) produce neurons in the striatum of rabbits (Luzzati et al., 2006) and in a mouse model of striatal degeneration (Luzzati et al., 2011b).

Here, we analysed striatal neurogenesis in the quinolinic acid (QA) lesion mouse model of Huntington's disease (Fan and Raymond, 2007). We show that such a lesion activates striatal astrocytes to produce neurons.

\section{RESULTS AND DISCUSSION}

At 5 weeks post-QA lesion (w.p.1.), numerous $\mathrm{DCX}^{+}$neuroblasts were present in the striatum and organised into clusters or as

\footnotetext{
'Department of Life Sciences and Systems Biology (DBIOS), University of Turin, Turin 10123, Italy. ${ }^{2}$ Neuroscience Institute Cavalieri Ottolenghi (NICO), Orbassano 10043 Italy. ${ }^{3}$ Departement of Biomedecin, University of Basel, Basel 4050, Switzerland. ${ }^{4}$ Department of Neuroscience Rita Levi-Montalcini, University of Turin, Turin 10126, Italy.

*These authors contributed equally to this work

${ }^{\ddagger}$ Authors for correspondence (paolo.peretto@unito.it; federico.luzzati@unito.it)
}

Received 14 August 2014; Accepted 14 January 2015 individual cells (Fig. 1A-E). As in other models of striatal neurogenesis (Liu et al., 2009; Luzzati et al., 2011b), these neuroblasts expressed SP8, a transcription factor typical of lateral/ caudal ganglionic eminence-derived interneurons (Ma et al., 2012; Waclaw et al., 2006), and some of them expressed NeuN (RBFOX3 Mouse Genome Informatics; data not shown) and attained complex morphologies (supplementary material Fig. S1). The clusters of $\mathrm{DCX}^{+}$cells were closely associated to clusters of cells expressing the proliferation marker Ki67 (MKI67 - Mouse Genome Informatics), with numerous cells colabelled for DCX (Fig. 1B,C,E).

Based on clustering and the differential expression of $\mathrm{Ki} 67$ and DCX we could define four striatal cell types that were induced by QA: clustered $\mathrm{Ki}^{+} 7^{+} / \mathrm{DCX}^{-}$cells (cK), clustered $\mathrm{Ki}^{+} / \mathrm{DCX}^{+}$cells (cKD), clustered $\mathrm{DCX}^{+} / \mathrm{Ki}^{-} 7^{-}$cells $(\mathrm{cD})$ and individual $\mathrm{DCX}^{+} /$ Ki67 ${ }^{-}$cells (iD) (Fig. 1C-F). The cK, cKD and cD cells appeared between 2 and 3 w.p.l. (2 versus 3 w.p.l., Tukey's post-hoc test: cK, $P=0.010$; cKD $P=0.005$; cD, $P=0.021$; Fig. $1 \mathrm{~F})$ and, although their number remained constant after 3 weeks (ANOVA: $\mathrm{cK}, F_{2,7}=2.464$, $\left.P=0.155 ; \mathrm{cKD}, F_{2,7}=0.383, P=0.695 ; \mathrm{cD}, F_{2,7}=0.419, P=0.673\right)$, at all time points a high proportion incorporated $\mathrm{BrdU}$ when injected 4 days before sacrifice (supplementary material Fig. S2A,D). This indicates that $\mathrm{cK}, \mathrm{cKD}$ and $\mathrm{cD}$ cells have a high turnover rate. By contrast, iD cells showed a delayed increase that peaked at 4 w.p.l. (Tukey's post-hoc test: 2 versus 3 weeks, $P=0.226$; 3 versus 4 weeks, $P=0.001$; Fig. $1 \mathrm{~F}$ ) accompanied by a reduction in the fraction of $\mathrm{BrdU}^{+}$cells over time (Tukey's post-hoc test: 3 versus 4 weeks, $P=0.031$; supplementary material Fig. S2C,D). Thus, this latter population appears later and has a lower turnover rate.

Interestingly, most cK cells expressed the TAP markers ASCL1 (Parras et al., 2004) and SOX9 (Cheng et al., 2009) (Fig. 1G-I; data not shown). Collectively, these data suggest that, as proposed in other models of striatal neurogenesis (Luzzati et al., 2006, 2011b), QA stimulates the appearance of TAP-like progenitors (cK cells) that give rise to neuroblasts that initially cluster ( $\mathrm{CKD}, \mathrm{cD}$ cells) and subsequently disperse as individual cells (iD cells). At 6 months after QA, striatal TAPs and neuroblasts were still present and could incorporate BrdU (supplementary material Fig. S3), suggesting that QA results in the long-term establishment of an intrastriatal neurogenic niche.

The induction of neurogenic potential in resident parenchymal cells was further supported by the appearance of self-renewing multipotent neurospherogenic cells in the striatum at 5 w.p.l. (supplementary material Fig. S4; data not shown). Interestingly, clusters of $\mathrm{cK}, \mathrm{cKD}$ and $\mathrm{cD}$ cells were generally closely associated to $\mathrm{GFAP}^{+}$astrocytes, which were occasionally proliferating, as assessed through both Ki67 and BrdU (supplementary material Fig. S5). Using hGFAP-GFP mice (Platel et al., 2009; Zhuo et al., 1997) we could establish that $\mathrm{GFP}^{+} / \mathrm{Ki}^{+} 7^{+}$proliferating astrocytes represented $8 \pm 3 \%$ of all cK cells at 5 w.p.l. (Fig. 1I-L). These observations support the contention that $\mathrm{cK}$ cells and their progeny originate from striatal astrocytes. 

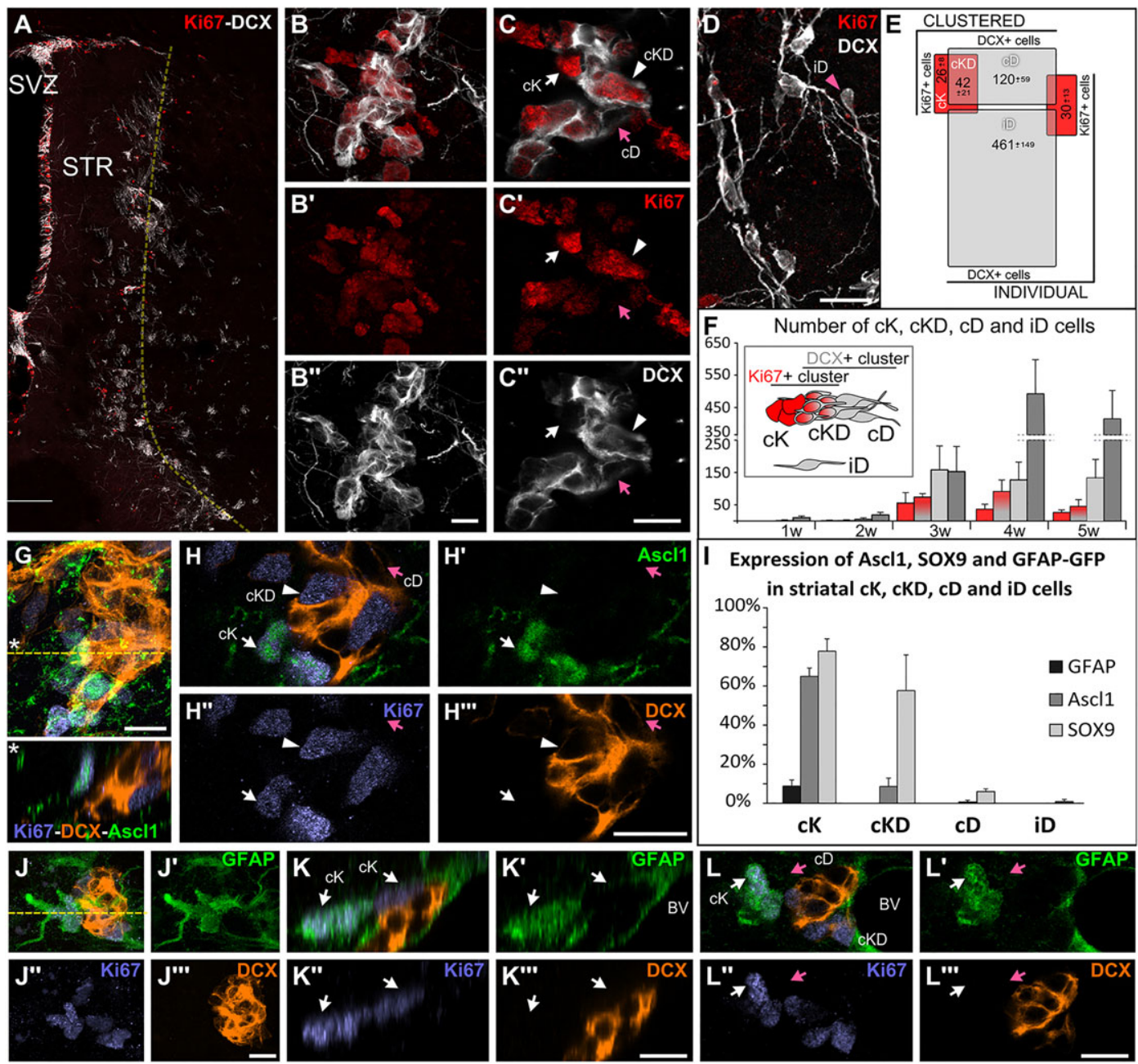

Fig. 1. $\mathrm{Ki}^{+} \mathrm{T}^{+}$and $\mathrm{DCX} \mathbf{C}^{+}$cells in the $\mathbf{5}$ w.p.I. striatum. (A) Coronal section showing striatal Ki67 ${ }^{+}$(red) and $\mathrm{DCX}{ }^{+}$(white) cells densely packed at the lesion border (yellow dashed line). (B-C $\left.\mathrm{C}^{\prime \prime}\right)$ z-projection (B-B") and single confocal plane (C-C") of a Ki67 $7^{+}$cluster partially overlapping with a DCX ${ }^{+}$cluster. (D) iD cells.

(E) Each square represents the number of individual (bottom right) or clustered (top left) DCX ${ }^{+}$(grey) and Ki67 (red) cells in the striatum at 5 w.p.l. The overlap of these populations is in dark red. (F) Number of $\mathrm{cK}, \mathrm{cKD}, \mathrm{cD}$ and iD cells in the striatum at 1, 2, 3, 4 and 5 w.p.I. A schematic view of the cell types is shown in the inset. (G-H'") z-projection (G), reslice (asterisk in G marks plane of section beneath) and single confocal plane $\left(\mathrm{H}^{\prime} \mathrm{H}^{\prime \prime \prime}\right)$ of a Ki67 ${ }^{+}$(violet) and DCX ${ }^{+}$(orange) cluster immunolabelled for ASCL1 (green). (I) Percentage of cK, cKD, cD and iD cells expressing GFAP-GFP, ASCL1 and SOX9. (J-L"') Z-projection (J-J"'), reslice (K-K'"', at yellow dashed line in J) and single confocal plane (L-L"') of a Ki67 ${ }^{+}$(violet) and DCX ${ }^{+}$(orange) cluster showing cK cells labelled for GFAP-GFP (green). Error bars indicate s.d. Scale bars: $200 \mu \mathrm{m}$ in A; $10 \mu \mathrm{m}$ in B-C", G-H"'; $20 \mu \mathrm{m}$ in D $10 \mu \mathrm{l}$ in J-L"'.

To explore this possibility further, we first performed cell fatemapping analysis using transgenic mice expressing the tamoxifeninducible recombinase $\mathrm{CreER}^{\mathrm{T} 2}$ under the control of diverse cell type-specific promoters, namely Glast (Slcla3 - Mouse Genome Informatics), nestin (Nes) and Ng2 (Cspg4 - Mouse Genome Informatics). GLAST is a pan-astrocytic marker (Dimou and Götz, 2014), whereas nestin more specifically associates with active neurogenic astrocytes (Codega et al., 2014) and some oligodendrocyte progenitors (Boda et al., 2015), while NG2 is specifically expressed by oligodendrocyte progenitors (Zhu et al., 2011). Accordingly, in intact animals 1 week after tamoxifen, recombined $\mathrm{YFP}^{+}$cells represented $44 \pm 3 \%$ of all $\mathrm{S} 100 \mathrm{~b}^{+}$striatal astrocytes in GLAST-CreER ${ }^{\mathrm{T} 2}$ mice and $10 \pm 6 \%$ in Nestin-CreER ${ }^{\mathrm{T} 2}$ animals. In the NG2-CreER ${ }^{\mathrm{T} 2}$ line, astrocytes were not targeted (supplementary material Fig. S6).

To determine whether cells expressing these genes are the source of intrastriatal TAPs and neuroblasts after lesion, tamoxifen was administered 1 week before QA (bQA). In addition, for each genotype a second group of animals was treated with tamoxifen at
4 w.p.l. (aQA) to identify possible injury-related changes in the phenotype of the neurogenic progenitors after their activation. In all cases animals were analysed at 5 w.p.l. (Fig. 2A). In NG2-CreER ${ }^{\mathrm{T} 2}$ animals, we never observed $\mathrm{DCX}^{+}$neuroblasts expressing the reporter YFP, either in the striatum or in the subventricular zoneolfactory bulb (SVZ-OB) system (data not shown), indicating that $\mathrm{NG}^{+}$cells are not neurogenic in our model. By contrast, in the SVZ of GLAST-CreER ${ }^{\mathrm{T} 2}$ and Nestin-CreER ${ }^{\mathrm{T} 2}$ animals, $\mathrm{YFP}^{+}$cells included putative TAPs $\left(\mathrm{Ki}^{+} / \mathrm{DCX}^{-}\right)$, proliferating $\left(\mathrm{DCX}^{+} /\right.$ $\left.\mathrm{Ki} 67^{+}\right)$and postmitotic $\left(\mathrm{DCX}^{+} / \mathrm{Ki}^{-} 7^{-}\right.$) neuroblasts (Fig. 2A-C). The percentage of $\mathrm{YFP}^{+}$cells did not differ between strains (supplementary material Table S1), indicating similar efficiency of nestin- or GLAST-driven recombination in SVZ neurogenic astrocytes.

In the striatum of GLAST-CreER ${ }^{\mathrm{T} 2} \mathrm{bQA}$ and aQA animals, YFP was expressed by $\mathrm{cK}, \mathrm{cKD}, \mathrm{cD}$ and $\mathrm{iD}$ cells, indicating that these cells originate from astrocytes that express GLAST both before and after the QA lesion (Fig. 2A,D,E; supplementary material Fig. S7A,B). $\mathrm{YFP}^{+}$postmitotic neuroblasts ( $\mathrm{CD}$ and $\mathrm{iD}$ ) were less numerous in the 

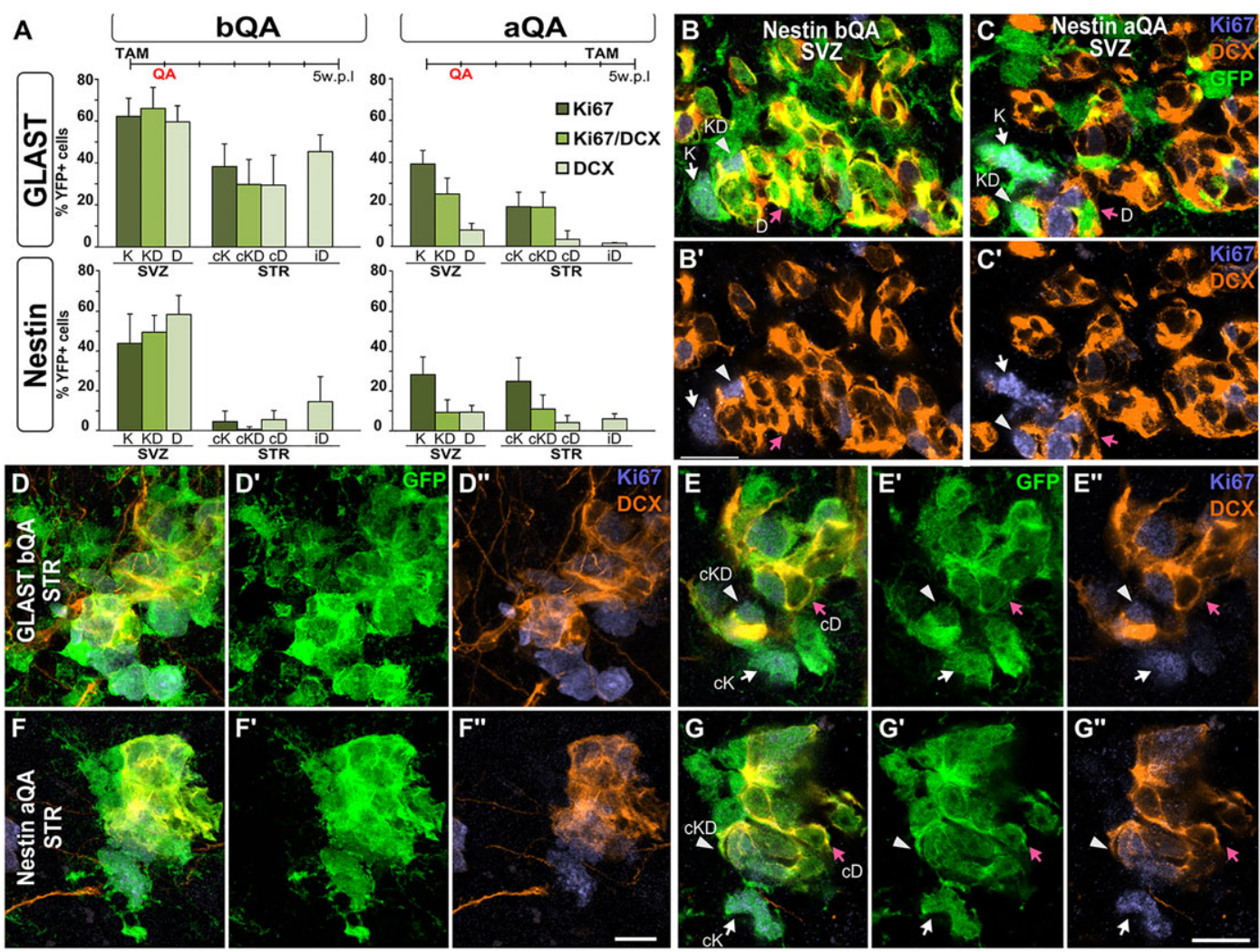

Fig. 2. Genetic lineage tracing. (A) Fraction of SVZ and striatal (STR) putative TAP cells (Ki67 ${ }^{+} ; \mathrm{K}$, cK), proliferating (Ki67 ${ }^{+} / \mathrm{DCX}{ }^{+} ; \mathrm{KD}$, $\left.\mathrm{cKD}\right)$ and post-mitotic $\left(\mathrm{DCX}^{+} ; \mathrm{D}, \mathrm{cD}, \mathrm{iD}\right.$ ) neuroblasts that expressed YFP in 5 w.p.l. GLAST-CreER ${ }^{\mathrm{T2}}$ (top row) and Nestin-CreER ${ }^{\mathrm{T} 2}$ (bottom row) animals that received TAM before (bQA, left column) or after (aQA, right column) lesion. Error bars indicate s.d. (B-C') Single confocal plane of the SVZ of a Nestin-CreER ${ }^{\mathrm{T} 2}$ bQA (B) and aQA (C) labelled for Ki67 (violet), DCX (orange) and GFP (green). (D-G") z-projections (D,F) and single confocal planes (E,G) of recombined Ki67 ${ }^{+} / D C X^{+}$clusters. Scale bars: $10 \mu \mathrm{m}$.

striatum of GLAST-CreER ${ }^{\mathrm{T} 2}$ aQA animals, but increased to levels comparable to those of $\mathrm{YFP}^{+}$proliferative cells (cK and $\left.\mathrm{cKD}\right)$ in the GLAST-CreER $^{\mathrm{T} 2}$ bQA animals (Fig. 2A; supplementary material Table S1A), further supporting that $\mathrm{cK}$ and $\mathrm{cKD}$ cells are early stages of the striatal neurogenic lineage. Three-dimensional reconstructions in GLAST-CreER ${ }^{\mathrm{T} 2}$ bQA animals indicated that the morphology of the $\mathrm{GFP}^{+} \mathrm{iD}$ cells was comparable to that of their $\mathrm{GFP}^{-}$counterparts (supplementary material Fig. S8). Thus, $\mathrm{GLAST}^{+}$astrocytes are the source of QA-induced intrastriatal TAPs and neuroblasts.

In Nestin-CreER ${ }^{\mathrm{T} 2} \mathrm{bQA}$ mice, $\mathrm{YFP}^{+}$cells corresponding to the striatal cell types (cK, cKD, cD and iD) were very rare (Fig. 2A). However, in Nestin-CreER ${ }^{\mathrm{T} 2}$ aQA animals, the levels of genetic labelling of $\mathrm{cK}$ and $\mathrm{cKD}$ cells were greatly increased and reached similar levels to those seen in GLAST-CreER ${ }^{\mathrm{T} 2}$ aQA animals (Fig. 2A,F,G; supplementary material Table S1C). Interestingly, although nestin was mostly absent from striatal astrocytes under normal conditions, several $\mathrm{YFP}^{+}$cells with astrocytic morphology appeared in Nestin-CreER ${ }^{\mathrm{T} 2}$ aQA animals (supplementary material Fig. S7C,D). These findings suggest that the resident GLAST ${ }^{+}$ striatal astrocytes upregulate the expression of nestin after lesion and generate $\mathrm{cK}, \mathrm{cKD}, \mathrm{cD}$ and $\mathrm{iD}$ cells.

To directly confirm both the striatal origin of the neurogenic progenitors and their astrocytic identity, we performed intrastriatal injections of either a GFP-tagged lentiviral vector (VSVG-GFP; $n=3$ ) or an adenoviral vector carrying Cre recombinase under the control of the mouse Gfap promoter (Ad:GFAP-Cre; $n=3$; Merkle et al., 2007) 1 week before QA lesion. Whereas VSVG-GFP showed broad cellular tropism (data not shown), injection of Ad: GFAP-Cre into the striatum of R26R reporter mice resulted in the expression of YFP almost exclusively in astrocytes (supplementary material Fig. S9). Only animals with no YFP staining in the SVZOB system were analysed. In both cases, at 5 w.p.l. serial section 3D reconstructions of the whole striatum revealed multiple examples of $\mathrm{YFP}^{+}$or $\mathrm{GFP}^{+} \mathrm{cK}, \mathrm{cKD}, \mathrm{cD}$ and iD cells (Figs 3 and 4). The morphology of these latter cells was consistent with that of $\mathrm{GFP}^{-} \mathrm{iD}$ cells (Fig. 4B-E). These data indicate that striatal astrocytes generate TAPs and neuroblasts after lesion.

Notably, in both our genetic and viral fate-mapping analyses about $85 \%$ of the striatal $\mathrm{Ki} 67^{+}$clusters exhibiting reporter expression were entirely composed of cells expressing YFP or GFP (Fig. 2D-G, Fig. 3C,D and Fig. 4F,G; see Materials and Methods for details), indicating that proliferative clusters originate mostly from the clonal expansion of a single striatal astrocytic progenitor.

SVZ progenitors have been shown to generate neuroblasts for the lesioned striatum (Liu et al., 2009). To examine whether these progenitors can further contribute to the intrastriatal TAPs, we injected Ad:GFAP-Cre or a TAT-Cre to respectively target the dorsolateral and the periventricular SVZ of R26R mice 1 week before QA. In the striatum of these animals at 5 w.p.l. we observed only a few iD cells expressing YFP (supplementary material Fig. S10), suggesting that striatal TAPs originate only from local astrocytes.

Taken together, these results indicate that some striatal astrocytes are quiescent neuronal progenitors that become activated after QA lesion. Like neurogenic astrocytes of other neurogenic niches, these cells upregulate nestin in their active state (Codega et al., 2014) and 

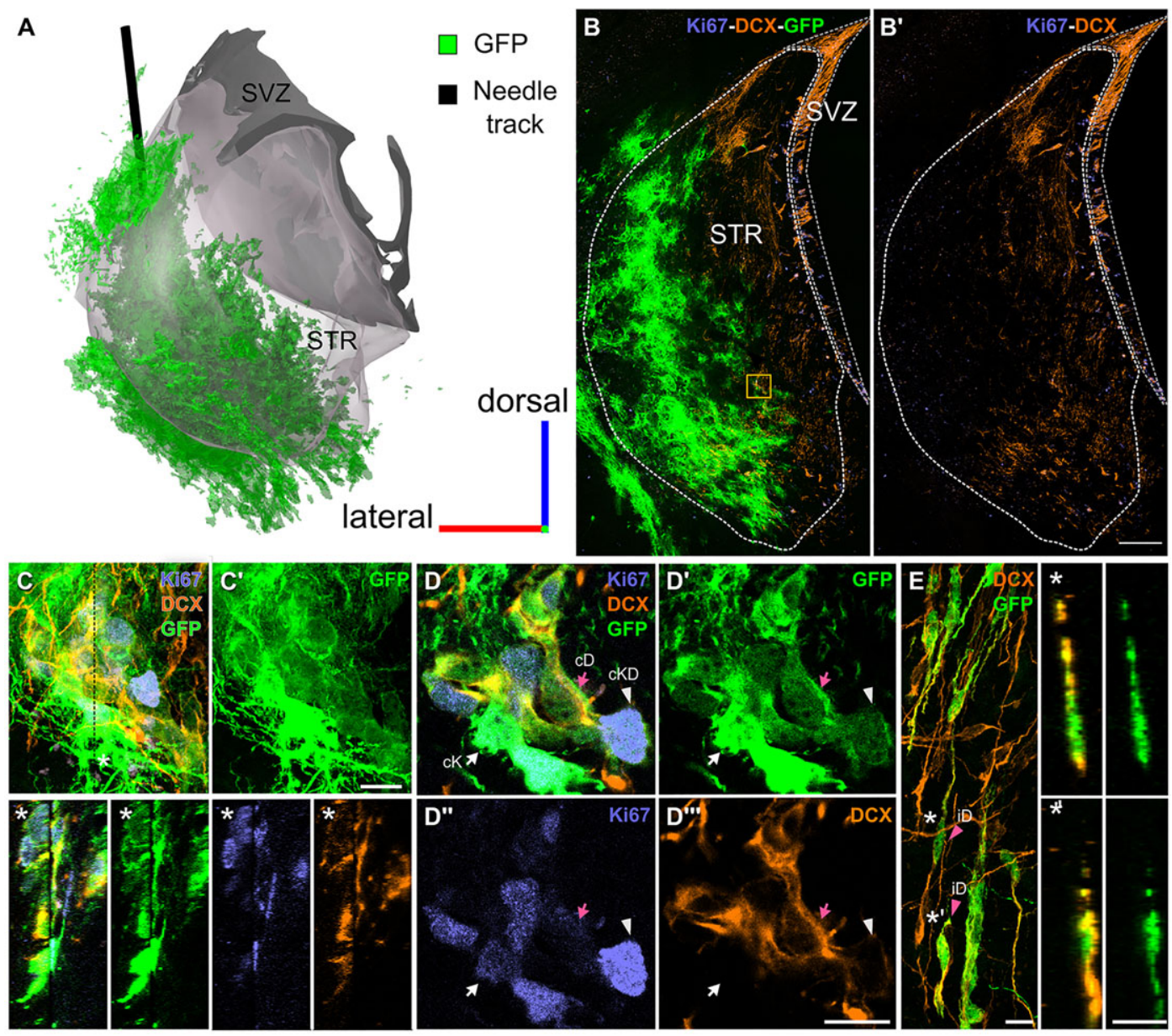

Fig. 3. Viral lineage tracing. (A) 3D reconstruction of SVZ (grey), striatum (transparent grey) and GFP staining (green) of a 5 w.p.l. animal injected with VSVG-GFP 1 week before QA. (B, B') Coronal section at the level of the injection site labelled for DCX (orange), Ki67 (white) and GFP (green). (C-D"') z-projection (C), reslice (asterisk, at black dotted line) and single confocal plane (D-D"') of a cluster (box in B) made by cK, cKD and cD cells reconstructed from two successive $50 \mu \mathrm{m}$ serial sections. (E) z-projection and reslices (asterisk and asterisk with prime) of GFP ${ }^{+}$iD cells. Scale bars: $200 \mu \mathrm{m}$ in B,B'; $10 \mu \mathrm{m}$ in C-E.

produce neurons through $\mathrm{ASCL1}^{+}$and $\mathrm{SOX9}^{+} \mathrm{TAPs}$ (Dimou and Götz, 2014; Fuentealba et al., 2012). However, striatal progenitors and/or their microenvironment may possess unique features that enable them to produce neurons in the brain parenchyma. Unravelling these features might help to unleash the full neurogenic potential of the adult brain, a fundamental prerequisite in order to design cell replacement therapies for brain repair. Interestingly, while this study was under revision the activation of neurogenic potential in striatal astrocytes was also demonstrated in a model of stroke (Magnusson et al., 2014) and under physiological conditions during guinea pig development (Luzzati et al., 2014). Thus, in contrast to SVZ and dentate gyrus neuronal progenitors, which are constitutively active, other populations of neurogenic astrocytes are activated only under specific conditions. The fate potential of these progenitors remains an important issue. Most neuroblasts generated in both the normal and lesioned striatum have a short life-span, but attain complex and specific morphologies (Luzzati et al., 2011a,b, 2014). These transient neurons might sustain a new type of adult brain plasticity that merits further exploration.

\section{MATERIALS AND METHODS}

\section{Animals}

All animal experiments were approved by the Italian Ministry of Health and the Bioethical Committee of the University of Turin. Experiments were performed on 8- to 12-week animals. C57BL/6 lesioned mice received two intraperitoneal injections (6 $\mathrm{h}$ apart) of 5-bromo-2-deoxyuridine (BrdU, Sigma-Aldrich; $50 \mathrm{mg} / \mathrm{kg}$ in $0.1 \mathrm{M}$ Tris $\mathrm{pH}$ 7.4) 4 days before sacrifice. Tamoxifen (TAM, Sigma-Aldrich T5648-1G) was dissolved in corn oil (Sigma-Aldrich C8267) and $2.5 \mathrm{mg}$ was administered by forced feeding (oral gavage) twice with a $24 \mathrm{~h}$ interval.

\section{Histology}

Animals were anesthetised with a ketamine (100 mg/kg ketavet, Gellini) and xylazine ( $33 \mathrm{mg} / \mathrm{kg}$ rompun, Bayer) solution and perfused with a solution of $4 \%$ paraformaldehyde (PFA) and 2\% picric acid (AnalytiCals, Carlo Erba 409302) in $0.1 \mathrm{M}$ sodium phosphate buffer (PB) $\mathrm{pH}$ 7.4. Brains were then post-fixed for $3 \mathrm{~h}$, cryoprotected in $30 \%$ sucrose (Fluka 84100 ) in $0.1 \mathrm{M} \mathrm{PB}$ $\mathrm{pH} 7.4$, embedded at $-80^{\circ} \mathrm{C}$ in Killik/OCT (Bio-Optica 05-9801), and cryostat sectioned in a series of $50 \mu \mathrm{m}$-thick sections.

\section{Generation of viral vectors and TAT-Cre}

VSVG-GFP vector stocks were produced by transient transfection of the transfer plasmid expressing eGFP under the control of the CMV promoter, the packaging plasmids pMDLg/pRRE and pRSV.REV, and the VSV envelope plasmid pMD2.VSV-G in HEK293T cells as described (Follenzi et al., 2000). Viral particles were purified and concentrated by ultracentrifugation as described (Dull et al., 1998). Vector titre on HeLa cells was $2 \times 10^{9} \mathrm{TU} / \mathrm{ml}$. The virus was then diluted $1 / 20$ in PBS containing $0.6 \%$ glucose and frozen.

Generation of Ad:GFAP-Cre virus was described previously (Merkle et al., 2014). Briefly, HEK293 cells were infected to produce replication-defective adenovirus, which was purified using the Fast-Trap 

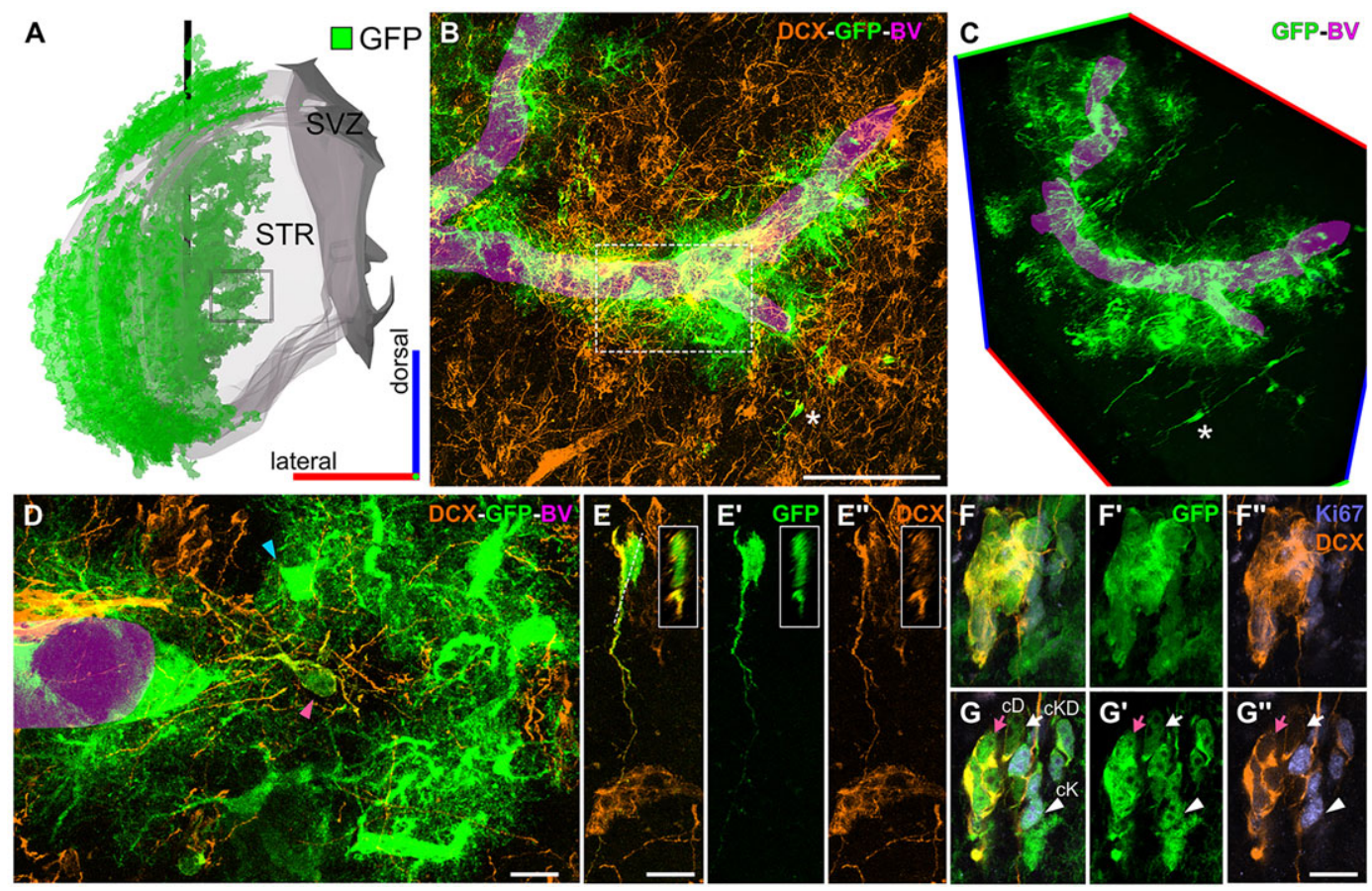

Fig. 4. Intrastriatal injection of Ad:GFAP-Cre. (A) 3D reconstruction of SVZ (grey), striatum (transparent grey), GFP staining (green) and needle track (black bar) of a 5 w.p.I. R26R-YFP animal injected with Ad:GFAP-Cre 1 week before QA. (B,C) z-projection (B) and perspective view (C) of a 3D reconstruction comprising four $50 \mu \mathrm{m}$-thick sections labelled with YFP (green) and DCX (orange) (box in A). Most YFP ${ }^{+}$cells are distributed around blood vessels (BV, magenta). However, some $\mathrm{DCX}^{+}$cells are also found deeper in the striatal parenchyma. (D) z-projection of a single $50 \mu \mathrm{m}$ section (box in B) showing YFP $/ \mathrm{DCX}^{+}$ (pink arrowhead) and $\mathrm{YFP}^{+} / \mathrm{DCX}^{-}$(blue arrowhead) cells near a blood vessel. (E-E") Higher magnification of an individual $\mathrm{YFP}^{+} / \mathrm{DCX}^{+}$cell, as indicated by the asterisk in B,C. The inset shows a reslice. (F-G") z-projection (F-F") and single confocal plane (G-G") of a cluster made by cK, cKD and cD cells. Scale bars: $100 \mu \mathrm{m}$ in $\mathrm{B} ; 10 \mu \mathrm{m}$ in D-G".

Adenovirus Purification and Concentration Kit (Millipore). The titre was $1 \times 10^{10}$ infectious particles $/ \mathrm{ml}$. TAT-Cre recombinant protein was produced as previously described (Peitz et al., 2002).

\section{Stereotaxic injections}

Mice were anesthetised with $0.3 \mathrm{ml} / \mathrm{kg}$ ketamine and $0.2 \mathrm{ml} / \mathrm{kg}$ xylazine, positioned in a stereotaxic apparatus (Stoelting) and injected with a pneumatic pressure injection apparatus (Picospritzer II, General Valve Corporation). Injection coordinates: QA (Sigma-Aldrich P6,320-4; $1 \mu 1$ diluted to $120 \mathrm{mM}$ in $0.1 \mathrm{MPB}$ ), $+0.1 \mathrm{~mm} \mathrm{AP},-2.1 \mathrm{~mm} \mathrm{ML}$ and $-2.6 \mathrm{~mm}$ DV; VSVG-GFP and Ad:GFAP-Cre, $0.8 \mathrm{~mm} \mathrm{AP,}-2.1 \mathrm{~mm} \mathrm{ML}$ and $-3.2 \mathrm{~mm} \mathrm{DV}$ ( $n=3$ for each vector). Ad:GFAP-Cre virions driving Cre recombinase expression in $\mathrm{GFAP}^{+}$cells were injected into R26YFP reporter mice. For both vectors, we analysed only animals in which the SVZ and OB were entirely free of reporter-positive cells. To target the SVZ, VSVG-GFP and TAT-Cre were injected respectively at $+1.2 \mathrm{~mm} \mathrm{AP},-1 \mathrm{~mm} \mathrm{ML}$ and $-1.3 \mathrm{~mm} \mathrm{DV}$ and at $+3 \mathrm{~mm} \mathrm{AP},-0.8 \mathrm{~mm} \mathrm{ML}$ and $-2.9 \mathrm{~mm} \mathrm{DV}$.

\section{Immunofluorescence}

Sections were incubated for $48 \mathrm{~h}$ at $4^{\circ} \mathrm{C}$ in $0.01 \mathrm{M}$ PBS pH 7.4 containing $2 \%$ Triton X-100, 1:100 normal donkey serum and primary antibodies (supplementary material Table S2). For BrdU staining, sections were preincubated in $2 \mathrm{M} \mathrm{HCl}$ for $30 \mathrm{~min}$ at $37^{\circ} \mathrm{C}$ and then rinsed in $0.1 \mathrm{M}$ borate buffer $\mathrm{pH}$ 8.5. Sections were incubated overnight with appropriate secondary antibodies (supplementary material Table S2) and coverslipped with antifade mounting medium Mowiol (4-88 reagent, Calbiochem 475904).

\section{Image processing and 3D reconstructions}

Images were processed using ImageJ (NIH) and Photoshop 7.0 (Adobe Systems). Confocal microscopy serial section 3D reconstructions were performed as described (Luzzati et al., 2011a). Briefly, images from each section were stitched in Fiji (Preibisch et al., 2009), aligned with Reconstruct 1.1 (Fiala, 2005) or with TracKEM2 (Cardona et al., 2010) and neurons were traced in NeuronStudio (Wearne et al., 2005). 3D models were rendered in Blender 2.6. (Blender Foundation) and Vaa3D (Peng et al., 2014).

\section{Acknowledgements}

We thank Claudio Giachino for critical reading of the manuscript; Rosanna Parlato and Ghunther Shutz for the Nestin-CreER ${ }^{T 2}$ transgenic line; Arturo Alvarez-Buylla for the Ad:GFAP-Cre plasmids; Elisa Vigna for VSVG-GFP plasmids; and Massimiliano Mazzone for TAT-Cre. This work is dedicated to the memory of Aldo Fasolo.

\section{Competing interests}

The authors declare no competing or financial interests.

\section{Author contributions}

F.L., P.P. and G.N. designed the experiments. A.B. contributed to the experimental design. F.L. and G.N. performed the experiments and analysed the data. A.C. contributed to the lineage-tracing study. S.T. and V.A. contributed to the stereological analyses. V.T. provided the Ad:GFAP-Cre viral vector. C.R. produced and tested the viral vectors. A.B. contributed to the neurosphere assays. F.L., P.P. and A.B. wrote the paper. V.T. critically commented on the paper.

\section{Funding}

This work was supported by Progetti di Ricerca di Interesse Nazionale (PRIN)Peretto $2010-2011$ and partly by PRIN 20107MSMA4 to A.B.

\section{Supplementary material}

Supplementary material available online at

http://dev.biologists.org/lookup/suppl/doi:10.1242/dev.116657/-/DC1

\section{References}

Bi, B., Salmaso, N., Komitova, M., Simonini, M. V., Silbereis, J., Cheng, E., Kim, J., Luft, S., Ment, L. R., Horvath, T. L. et al. (2011). Cortical glial fibrillary acidic protein-positive cells generate neurons after perinatal hypoxic injury. J. Neurosci. 31, 9205-9221.

Boda, E., Di Maria, S., Rosa, P., Taylor, V., Abbracchio, M. P. and Buffo, A. (2015). Early phenotypic asymmetry of sister oligodendrocyte progenitor cells after mitosis and its modulation by aging and extrinsic factors. Glia 63, 271-286. 
Buffo, A., Rite, I., Tripathi, P., Lepier, A., Colak, D., Horn, A.-P., Mori, T. and Gotz, M. (2008). Origin and progeny of reactive gliosis: a source of multipotent cells in the injured brain. Proc. Natl. Acad. Sci. USA 105, 3581-3586.

Cardona, A., Saalfeld, S., Preibisch, S., Schmid, B., Cheng, A., Pulokas, J., Tomancak, P. and Hartenstein, V. (2010). An integrated micro- and macroarchitectural analysis of the Drosophila brain by computer-assisted serial section electron microscopy. PLoS Biol. 8, e1000502.

Cheng, L.-C., Pastrana, E., Tavazoie, M. and Doetsch, F. (2009). miR-124 regulates adult neurogenesis in the subventricular zone stem cell niche. Nat. Neurosci. 12, 399-408.

Codega, P., Silva-Vargas, V., Paul, A., Maldonado-Soto, A. R., Deleo, A. M., Pastrana, E. and Doetsch, F. (2014). Prospective identification and purification of quiescent adult neural stem cells from their in vivo niche. Neuron 82, 545-559.

Corsini, N. S., Sancho-Martinez, I., Laudenklos, S., Glagow, D., Kumar, S., Letellier, E., Koch, P., Teodorczyk, M., Kleber, S., Klussmann, S. et al. (2009). The death receptor CD95 activates adult neural stem cells for working memory formation and brain repair. Cell Stem Cell 5, 178-190.

Dimou, L. and Götz, M. (2014). Glial cells as progenitors and stem cells: new roles in the healthy and diseased brain. Physiol. Rev. 94, 709-737.

Dull, T., Zufferey, R., Kelly, M., Mandel, R. J., Nguyen, M., Trono, D. and Naldini, L. (1998). A third-generation lentivirus vector with a conditional packaging system. J. Virol. 72, 8463-8471.

Fan, M. M. Y. and Raymond, L. A. (2007). N-methyl-D-aspartate (NMDA) receptor function and excitotoxicity in Huntington's disease. Prog. Neurobiol. 81, 272-293.

Fiala, J. C. (2005). Reconstruct: a free editor for serial section microscopy. J. Microsc. 218, 52-61.

Follenzi, A., Ailles, L. E., Bakovic, S., Geuna, M. and Naldini, L. (2000). Gene transfer by lentiviral vectors is limited by nuclear translocation and rescued by HIV-1 pol sequences. Nat. Genet. 25, 217-222.

Fuentealba, L. C., Obernier, K. and Alvarez-Buylla, A. (2012). Adult neural stem cells bridge their niche. Cell Stem Cell 10, 698-708.

Lim, D. A., Tramontin, A. D., Trevejo, J. M., Herrera, D. G., García-Verdugo, J. M. and Alvarez-Buylla, A. (2000). Noggin antagonizes BMP signaling to create a niche for adult neurogenesis. Neuron 28, 713-726.

Liu, X. S., Chopp, M., Zhang, R. L., Hozeska-Solgot, A., Gregg, S. C., Buller, B., Lu, M. and Zhang, Z. G. (2009). Angiopoietin 2 mediates the differentiation and migration of neural progenitor cells in the subventricular zone after stroke. J. Biol. Chem. 284, 22680-22689.

Lowenstein, P. R. and Castro, M. G. (2003). Inflammation and adaptive immune responses to adenoviral vectors injected into the brain: peculiarities, mechanisms, and consequences. Gene Ther. 10, 946-954.

Luzzati, F., De Marchis, S., Fasolo, A. and Peretto, P. (2006). Neurogenesis in the caudate nucleus of the adult rabbit. J. Neurosci. 26, 609-621.

Luzzati, F., Fasolo, A. and Peretto, P. (2011a). Combining confocal laser scanning microscopy with serial section reconstruction in the study of adult neurogenesis. Front. Neurosci. 5, 70.

Luzzati, F., De Marchis, S., Parlato, R., Gribaudo, S., Schütz, G., Fasolo, A. and Peretto, P. (2011b). New striatal neurons in a mouse model of progressive striatal degeneration are generated in both the subventricular zone and the striatal parenchyma. PLoS ONE 6, e25088.

Luzzati, F., Nato, G., Oboti, L., Vigna, E., Rolando, C., Armentano, M., Bonfanti, L., Fasolo, A. and Peretto P. (2014). Quiescent neuronal progenitors are activated in the juvenile guinea pig lateral striatum and give rise to transient neurons. Development 141, 4065-4075.

Ma, T., Zhang, Q., Cai, Y., You, Y., Rubenstein, J. L. R. and Yang, Z. (2012). A subpopulation of dorsal lateral/caudal ganglionic eminence-derived neocortical interneurons expresses the transcription factor Sp8. Cereb. Cortex 22, $2120-2130$
Magnusson, J. P., Göritz, C., Tatarishvili, J., Dias D. O., Smith E. M. K., Lindvall, O., Kokaia, Z. and Frisén J. (2014). A latent neurogenic program in astrocytes regulated by Notch signaling in the mouse. Science 346, 237-241.

Merkle, F. T., Mirzadeh, Z. and Alvarez-Buylla, A. (2007). Mosaic organization of neural stem cells in the adult brain. Science 317, 381-384.

Merkle, F. T., Fuentealba, L. C., Sanders, T. A., Magno, L., Kessaris, N. and Alvarez-Buylla, A. (2014). Adult neural stem cells in distinct microdomains generate previously unknown interneuron types. Nat. Neurosci. 17, 207-214.

Mori, T., Tanaka, K., Buffo, A., Wurst, W., Kühn, R. and Götz, M. (2006). Inducible gene deletion in astroglia and radial glia-a valuable tool for functional and lineage analysis. Glia 54, 21-34.

Ninkovic, J. and Götz, M. (2013). Fate specification in the adult brain-lessons for eliciting neurogenesis from glial cells. Bioessays 35, 242-252.

Niu, W., Zang, T., Zou, Y., Fang, S., Smith, D. K., Bachoo, R. and Zhang, C.-L. (2013). In vivo reprogramming of astrocytes to neuroblasts in the adult brain. Nat Cell Biol. 15, 1164-1175.

Peitz, M., Pfannkuche, K., Rajewsky, K. and Edenhofer, F. (2002). Ability of the hydrophobic FGF and basic TAT peptides to promote cellular uptake of recombinant Cre recombinase: a tool for efficient genetic engineering of mammalian genomes. Proc. Natl. Acad. Sci. USA 99, 4489-4494.

Peng, H., Bria, A., Zhou, Z., lannello, G. and Long, F. (2014). Extensible visualization and analysis for multidimensional images using Vaa3D. Nat. Protoc. 9, 193-208.

Ohira, K., Furuta, T., Hioki, H., Nakamura, K. C., Kuramoto, E., Tanaka, Y., Funatsu, N., Shimizu, K., Oishi, T., Hayashi, M. et al. (2010). Ischemia-induced neurogenesis of neocortical layer 1 progenitor cells. Nat. Neurosci. 13, 173-179.

Parras, C. M., Galli, R., Britz, O., Soares, S., Galichet, C., Battiste, J., Johnson, J. E., Nakafuku, M., Vescovi, A. and Guillemot, F. (2004). Mash1 specifies neurons and oligodendrocytes in the postnatal brain. EMBO J. 23, 4495-4505

Pastrana, E., Cheng, L.-C. and Doetsch, F. (2009). Simultaneous prospective purification of adult subventricular zone neural stem cells and their progeny. Proc. Natl. Acad. Sci. USA 106, 6387-6392.

Platel, J.-C., Gordon, V., Heintz, T. and Bordey, A. (2009). GFAP-GFP neural progenitors are antigenically homogeneous and anchored in their enclosed mosaic niche. Glia 57, 66-78

Preibisch, S., Saalfeld, S. and Tomancak, P. (2009). Globally optimal stitching of tiled 3D microscopic image acquisitions. Bioinformatics 25, 1463-1465.

Shihabuddin, L. S., Horner, P. J., Ray, J. and Gage, F. H. (2000). Adult spinal cord stem cells generate neurons after transplantation in the adult dentate gyrus. J. Neurosci. 20, 8727-8735.

Sirko, S., Behrendt, G., Johansson, P. A., Tripathi, P., Costa, M. R., Bek, S. Heinrich, C., Tiedt, S., Colak, D., Dichgans, M. et al. (2013). Reactive glia in the injured brain acquire stem cell properties in response to sonic hedgehog glia. Cell Stem Cell 12, 426-439.

Srinivas, S., Watanabe, T., Lin, C.-S., William, C. M., Tanabe, Y., Jessell, T. M. and Costantini, F. (2001). Cre reporter strains produced by targeted insertion of EYFP and ECFP into the ROSA26 locus. BMC Dev. Biol. 1, 4.

Waclaw, R. R., Allen, Z. J., Bell, S. M., Erdélyi, F., Szabó, G., Potter, S. S. and Campbell, K. (2006). The zinc finger transcription factor Sp8 regulates the generation and diversity of olfactory bulb interneurons. Neuron 49, 503-516.

Wearne, S. L., Rodriguez, A., Ehlenberger, D. B., Rocher, A. B., Henderson, S. C. and Hof, P. R. (2005). New Techniques for imaging, digitization and analysis of three-dimensional neural morphology on multiple scales. Neuroscience 136 $661-680$

Zhu, X., Hill, R. A., Dietrich, D., Komitova, M., Suzuki, R. and Nishiyama, A (2011). Age-dependent fate and lineage restriction of single NG2 cells. Development 138, 745-753.

Zhuo, L., Sun, B., Zhang, C. L., Fine, A., Chiu, S. Y. and Messing, A. (1997). Live astrocytes visualized by green fluorescent protein in transgenic mice. Dev. Biol. 187, 36-42. 